\title{
Figure-of-Eight Wire Sternal Closure Technique Can Reduce Post-Open Cardiovascular Surgery Chest Re- Exploration and Pain Scores in Diabetic Patients with Severe Obesity (Body Mass Index: 35-40)
}

\author{
Seyed Jalil Mirhosseini, ${ }^{1,}$, Sadegh Ali-Hassan-Sayegh ${ }^{2}$, \\ Seyed Mohammad Yousof Mostafavi-Pour-Manshadi ${ }^{2,3}$, Nafiseh Naderi, ${ }^{2,3}$, \\ Maryam Hadibarhaghtalab ${ }^{4}$, Mohammad Reza Lotfaliani ${ }^{2}$ \\ ${ }^{1}$ Department of Cardiac Surgery, Afshar Hospital, Shahid Sadoughi University of Medical Sciences and Health Services, Yazd, Iran \\ ${ }^{2}$ Yazd Cardiovascular Research Center, Afshar Hospital, Shahid Sadoughi University of Medical Sciences and Health Services, Yazd, Iran \\ ${ }^{3}$ Department of Medicine, Ali ben Abitalib Medical College, Islamic Azad University, Yazd, Iran \\ ${ }^{4}$ Non-Communicable Diseases Research Center, Fasa University of Medical Sciences, Fasa, Iran
}

\section{Email address:}

mirhosseinidr@gmail.com (S. J. Mirhosseini)

\section{To cite this article:}

Seyed Jalil Mirhosseini, Sadegh Ali-Hassan-Sayegh, Seyed Mohammad Yousof Mostafavi-Pour-Manshadi, Nafiseh Naderi, Maryam Hadibarhaghtalab, Mohammad Reza Lotfaliani. Figure-of-Eight Wire Sternal Closure Technique Can Reduce Post-Open Cardiovascular Surgery Chest Re-Exploration and Pain Scores in Diabetic Patients with Severe Obesity (Body Mass Index: 35-40). International Journal of Clinical and Experimental Medical Sciences. Vol. 1, No. 3, 2015, pp. 38-41. doi: 10.11648/j.ijcems.20150103.12

\begin{abstract}
Background. Postoperative sternal instability can be associated with the density of bone and type of wire closure. Sternal instability is a serious complication, especially in diabetic obese patients. This category of patients is susceptible to activation of inflammation and inflammatory and/or infective involvement of the sternum after sternotomy. This study was designed to evaluate the comparison of safety and efficacy of figure-of-eight with simple wire sternal closure technique in high-risk diabetic severe obese patients [Body mass index (BMI): 35-40]. Methods and materials. This study, conducted on 80 diabetic patients with severe obesity (BMI: 35-40) undergoing elective off-pump coronary artery bypass graft (CABG) surgery in duration from the first day to two weeks after surgery. Sternal wire closure techniques are divided into two groups, group A $(n=40)$, patients undergoing simple wire closure and group $B(n=40)$, patients undergoing figure-of-eight wire closure. Results. The average age of patients was $64.7 \pm 13.2$ years. According to the patient self-rated numeric pain intensity scale, the pain of sternum related to existing of wire closure was classified to mild [(group A: n=14), (group B: n=17)], moderate [(group A: n=14), (group B: 19)], and severe [(group A: n=12), (group B: 4)]. Postoperative pain scores had significant higher difference in simple closure than figure-of-eight technique. From all patients, three cases with figure-of-eight and seven cases with simple wire closure had chest re-exploration after surgery. Chest re-exploration in group of figure-of-eight was lower than group of simple closure significantly $(p=0.03)$. Postoperative utilization of inotropic agents, mediastinitis, cerebrovascular accident (CVA) and atrial fibrillation (AF) had not significant differences between both techniques. Conclusion. These findings show that figure-of-eight sternal wire closure technique can reduce postoperative pain scores and chest re-exploration significantly in diabetic patients with severe obesity.
\end{abstract}

Keywords: Figure-of-Eight Wire Sternal Closure, Obesity, Diabetic Patients, Off Pump CABG

\section{Introduction}

Coronary artery bypass graft (CABG) surgery is a common cardiac surgery in the world that has early and late surgical complications compared to other operations. ${ }^{1}$ Sternal dehiscence following median sternotomy is one of important complications after open-heart surgery. Sternal dehiscence is divided into non-infectious (this type is usually due to sternal wire cut-through that is a sterile dehiscence) or infectious (deep sternal wound infection). The most common risk factors for sternal surgical complications are included advanced age, abnormal body mass index, diabetes mellitus, smoking, chronic obstructive pulmonary disease (COPD), and prolonged 
duration of cardiopulmonary bypass. ${ }^{2,3}$ Mediastinitis and sternal dehiscence occur in $0.3-5 \%$ of all patients undergoing heart surgery and are associated with a mortality rate of 14$40 \%$. Inadequate sternal closure can lead to sternal instability and early, moderate and late surgical complications. ${ }^{4}$ Sternal instability following first days after surgery can be associated with the density of bone and type of wire closure and other factors. Sternal instability is a serious complication, especially in diabetic obese patients. ${ }^{3}$ This category of patients is susceptible to activation of inflammation and involvement of sternum after sternotomy. ${ }^{5}$ Previous studies have shown that utilization of more wires for closing of sternum can increase wound sternal infections, therefore, selection of optimal closure technique with less wires may improve postoperative outcomes. ${ }^{6}$ Currently, the most prevalent techniques for closing of sternum are interrupted simple wire and figure-ofeight wire technique. This study was designed to compare efficacy of figure-of-eight versus simple wire sternal closure in diabetic patients with severe obesity (BMI: 35-40) who are undergoing elective open-heart surgery. Advantages of this study compared with previous clinical papers are combination of diabetes mellitus and severe obesity and heart failure with ejection fraction less than $35 \%$ in all participants. In addition to these, type of open-heart operation was elective off pump CABG in our study.

\section{Methods and Materials}

This randomized double blind clinical trial was approved by the ethics committee in our university. After receiving the written consent from patients $(n=80)$, they participated voluntarily in off-pump CABG in Afshar cardiovascular surgery center of Yazd, Iran from February 2010 to March 2013. Each patient who had a history of re-operation and sternal fractures was excluded from our study. All of the operations were performed by a skilled cardiothoracic surgeon. Eighty diabetic patients with severe obesity (BMI: 35-40) undergoing elective off-pump CABG was evaluated in duration from the first day to two weeks after surgery. Sternal wire closure techniques were divided into two groups, group A $(\mathrm{n}=40)$, patients undergoing simple wire closure, and group B $(n=40)$, patients undergoing figure-ofeight wire closure. Stainless-steel No.5 wire was used for the closing of the sternum. According to a Turkish study about sternal closure technique, four figure-of-eight sutures were placed one cephalically at the manubrium and three in the body of the sternum. Simple wire technique was included the placement of two or three simple wire sutures at the manubrium and at least six parasternal simple wire sutures at the body of sternum. ${ }^{2}$ The demographic data and preoperative risk factors such as COPD, hypertension, ejection fraction, renal disease (creatinine level higher than normal range), risk factors during surgery (duration of cardiopulmonary bypass) and outcomes such as mortality, postoperative atrial fibrillation and cerebrovascular accident, prolonged intensive care unit (ICU), use of inotropic, and intra-aortic balloon pump (IABP) were recorded for all patients. According to the patient self-rated numeric pain intensity scale, the diagnosis and evaluation of pain were based on structured interview with the patient that was included the severity, location, quality, duration, course, and timing of the pain, as well as factors that make the pain better or worse. In some situations, the patient was asked to estimate the severity of his or her pain by pointing to a spot on a scale with numbers from 0 to 10 , with 0 , representing no pain, and with 10 , representing the worst imaginable pain. Our data were analyzed by SPSS15 software. Student T test, Mann Whitney U test, ANOVA, Chi-square and the Fisher exact test were used for variables. Any $p$-value $<0.05$ was considered as statistical significant.

\section{Results}

Eighty diabetic patients with severe obesity (BMI: 35-40) undergoing elective off pump CABG enrolled in this study. The average age of patients was $64.7 \pm 13.2$ years. From all participants, 51 cases $(63.7 \%)$ were male and 29 cases $(36.5 \%)$ were female. The two groups were similar in terms of age, sex and body mass index distribution. The prevalence of risk factors was as following, hypertension (36.25\%), hyperlipidemia (41.25\%), and cigarette smoking (50\%).

Table 1. Demographic characteristics of both groups.

\begin{tabular}{llll}
\hline Variables & Simple wire closure $(\mathbf{n}=\mathbf{4 0})$ & Figure of eight wire closure $(\mathbf{n}=\mathbf{4 0})$ & P-value \\
\hline Age $($ year) & $64 \pm 9.82$ & $63.2 \pm 11.48$ & 0.8 \\
Sex $(\mathrm{M} / \mathrm{F})(n)$ & $27 / 13$ & $24 / 16$ & 0.6 \\
HTN $[n(\%)]$ & $13(32.5 \%)$ & $16(40 \%)$ & 0.8 \\
Cigarette smoking $[n(\%)]$ & $21(52.5 \%)$ & $19(47.5 \%)$ & 0.7 \\
HLP $[n(\%)]$ & $15(37.5 \%)$ & $18(45 \%)$ & 0.7 \\
Advanced COPD $[n(\%)]$ & $11(27.5 \%)$ & $13(23.5 \%)$ & 0.8 \\
\hline
\end{tabular}

Data presented as mean \pm standard deviation, HTN: Hypertension, HLP: Hyperlipidemia, COPD: Chronic obstructive pulmonary disease

In patients with simple wire closure (group A), there were 3 cases $(7.5 \%)$ with one vessel, 6 cases $(15 \%)$ with two vessels, 24 cases $(60 \%)$ with three vessels, and 7 cases $(17.5 \%)$ suffering from left main coronary artery disease. In figure-of-eight group (group B), there were 4 cases (10\%) with one vessel, 8 cases $(20 \%)$ with two vessels, 23 cases
$(57.5 \%)$ with three vessels, and 5 cases $(12.5 \%)$ suffering from left main coronary artery disease. According to the patient self-rated numeric pain intensity scale, the pain of sternum related to existing of wire closure was classified to, mild [(group A: n=14), (group B: $n=17)$ ], moderate [(group A: $\mathrm{n}=14$ ), (group B: 19)], and severe [(group A: n=12), (group B: 
4)]. Postoperative pain scores had significant higher difference in simple closure than figure-of-eight technique $(p=0.03)$. Sternal dehiscence occurred in 2 cases, one case $(2.5 \%)$ in figure-of-eight closure and 1 case $(2.5 \%)$ in simple technique. There was no significant difference about sternal dehiscence between both groups $(p=1.0)$. The outcomes of both groups have been presented in Table 2 .

Table 2. Postoperative outcomes in both groups.

\begin{tabular}{|c|c|c|c|}
\hline Variables & Simple wire closure $(n=40)$ & Figure of eight wire closure $(n=40)$ & P-value \\
\hline Sternal dehiscence $[\mathrm{n}(\%)]$ & $1(2.5 \%)$ & $1(2.5 \%)$ & 1.0 \\
\hline Rewiring [n (\%)] & $2(5 \%)$ & $1(2.5 \%)$ & 0.8 \\
\hline IABP use $[\mathrm{n}(\%)]$ & $2(5 \%)$ & $1(2.5 \%)$ & 0.8 \\
\hline Inotropic use $[\mathrm{n}(\%)]$ & $10(25 \%)$ & $13(32.5 \%)$ & 0.6 \\
\hline Mediastinitis [n (\%)] & $3(7.5 \%)$ & $2(5 \%)$ & 0.8 \\
\hline CVA $[\mathrm{n}(\%)]$ & $1(2.5 \%)$ & $0(0 \%)$ & 0.9 \\
\hline $\mathrm{AF}[\mathrm{n}(\%)]$ & $9(22.5 \%)$ & $11(27.5 \%)$ & 0.7 \\
\hline
\end{tabular}

IABP: Intra-aortic balloon pump, CVA: Cerebrovascular accident, AF: Atrial fibrillation

Rewiring was performed in two cases $(5 \%)$ and one case $(2.5 \%)$ in group A and group B, respectively. Figure-of-eight technique versus simple technique could not decrease postoperative rewiring in high-risk patients significantly $(p=0.07)$. Proportion of patients required intra-aortic balloon pump and prolonged intensive care unit more than three days were similar in both groups. Postoperative utilization of inotropic agents (Group A: 25\% vs. Group B: 32.5\%), CVA (Group A: 2.5\% vs. Group B: 0\%), mediastinitis (Group A: 7.5\% vs. Group B: $5 \%$ ) and AF (Group A: $22.5 \%$ vs. Group B: $27.5 \%$ ) had not significant differences between both techniques. From all patients, three cases with figure-of-eight and seven cases with simple wire closure had chest reexploration due to major bleeding after surgery. Chest reexploration because of major bleeding in group of figure-ofeight was lower than group of simple closure significantly $(p=0.03)$.

Early mortality after the operation occurred in three cases $(3.75 \%)$ in hospital, 1 case in group of simple closure and two cases in group of figure-of-eight. There was no relationship between the type of techniques and early mortality.

\section{Discussion}

Early postoperative sternal instability can be associated with the density of bone and type of wire closure. ${ }^{2,3}$ Sternal instability is a serious complication, especially in diabetic obese patients. This category of patients is susceptible to activation of inflammation, inflammatory and/or infective involvement of the sternum after sternotomy. ${ }^{3,4}$ Our study shows that sternal dehiscence had not significant difference between both groups. In a study conducted by Tekümit et al., indicated that simple wire and figure-of-eight techniques have similar findings in prognosis sternal dehiscence in patients undergoing on-pump coronary artery bypass graft surgery. ${ }^{2}$ This study was in line with our study about sternal dehiscence, however, the type of revascularization coronary surgery in our study was offpump CABG surgery. Olbrecht et al. and Tekümit et al. reported that incidences of sternal dehiscence were $0.39 \%$ and $1.46 \%$, respectively. ${ }^{2,7}$ Incidence of sternal dehiscence in our study was $2.5 \%$. It is clear that patients with important risk factors such as diabetes and severe obesity affect sternal dehiscence. Our study focused on severity of postoperative pain, according to the self-rated numeric pain intensity scale between both groups. Figure-of-eight technique could decrease pain scores significantly compared to simple wire closure. Previous studies reported an association between utilization of several closure techniques and scores of postoperative pain. Sternal reconstruction after median sternotomy using rigid fixation with plates may decrease postoperative pain compared to wire. $^{8}$ A study carried out by Tekümit et al., showed that prolonged intensive care unit (ICU) stay, inotropic use, intra-aortic balloon pump (IABP) use, chest re-exploration, postoperative CVA and mortality had not significant differences in figure-of-eight and simple wire technique. ${ }^{2}$ Our findings are in line with this study, however, chest reexploration in group of figure-of-eight was lower than group of simple closure significantly. Aykut et al. conducted a study about figure-of-eight versus prophylactic sternal weave closure of median sternotomy in diabetic obese patients undergoing coronary artery bypass grafting. They reported that prophylactic sternal weave closure compare to figure-eight closure of sternotomy might reduce morbidity result from sternal dehiscence in high-risk patients undergoing $\mathrm{CABG}^{9}{ }^{9}$ Our findings indicate that mortality rates after simple and figure-of-eight closure were similar and had no significant difference. Ramzisham et al. suggested that figure-of-eight sternal closure is equally effective as simple wire suturing in preventing adverse outcomes of sternal dehiscence. ${ }^{10}$ Vular et al. showed that sternal closure reinforced with rib heads technique is extremely efficient and twenty-two patients from twentythree ones whom were treated with this technique survived $^{11}$ while our study showed that figure-eight and simple technique are more related to the risk of morbidity and mortality. ${ }^{11}$ Not accessing of association between bone healing and pain scores or complications, and not analyzing of the effect of pain severity on the activity or quality of life were limitations of our study. Finally, we concluded that 
figure-of-eight can reduce postoperative pain scores and chest re-exploration significantly in diabetic patients with severe obesity (BMI: 35-40) undergoing off-pump CABG surgery.

\section{Conflict of Interest}

None Declared

\section{References}

[1] Sá MP, Ferraz PE, Escobar RR, et al. Off-pump versus onpump coronary artery bypass surgery: meta-analysis and meta-regression of 13,524 patients from randomized trials. Rev Bras Cir Cardiovasc. 2012; 27:631-641.

[2] Tekümit H, Cenal AR, Tataroğlu C, Uzun K, Akinci E. Comparison of figure-of-eight and simple wire sternal closure techniques in patients with non-microbial sternal dehiscence. Anadolu Kardiyol Derg. 2009;9:411-416.

[3] Molina JE, Lew RS, Hyland KJ. Postoperative sternal dehiscence in obese patients: incidence and prevention. Ann Thorac Surg. 2004;78:912-917.

[4] Kamiya H, Al-maisary SS, Akhyari P, et al. The number of wires for sternal closure has a significant influence on sternal complications in high-risk patients. Interact Cardiovasc Thorac Surg. 2012;15:665-670.
[5] Cullen A, Ferguson A. Perioperative management of the severely obese patient: a selective pathophysiological review. Can J Anaesth. 2012;59:974-996.

[6] Shaikhrezai K, Robertson FL, Anderson SE, Slight RD, Brackenbury ET. Does the number of wires used to close a sternotomy have an impact on deep sternal wound infection? Interact Cardiovasc Thorac Surg. 2012;15:219-222.

[7] Olbrecht VA, Barreiro CJ, Bonde PN, et al. Clinical outcomes of noninfectious sternal dehiscence after median sternotomy. Ann Thorac Surg. 2006;82:902-907.

[8] Raman J, Lehmann S, Zehr K, et al. Sternal closure with rigid plate fixation versus wire closure: a randomized controlled multicenter trial. Ann Thorac Surg. 2012;94:1854-1861.

[9] Aykut K, Celik B, Acikel U. Figure-of-eight versus prophylactic sternal weave closure of median sternotomy in diabetic obese patients undergoing coronary artery bypass grafting. Ann Thorac Surg. 2011;92:638-641.

[10] Ramzisham AR, Raflis AR, Khairulasri MG, Ooi Su Min J, Fikri AM, Zamrin MD. Figure-of-eight vs. interrupted sternal wire closure of median sternotomy. Asian Cardiovasc Thorac Ann. 2009;17:587-591.

[11] Vural AH, Yalçinkaya S, Türk $\mathrm{T}$, et al. Sternal closure reinforced with rib heads: a novel technique for prevention and treatment of sternal dehiscence. Heart Surg Forum. 2007;10:E397-400. 